\title{
Recent developments and challenges in mouse models of Chikungunya virus infection
}
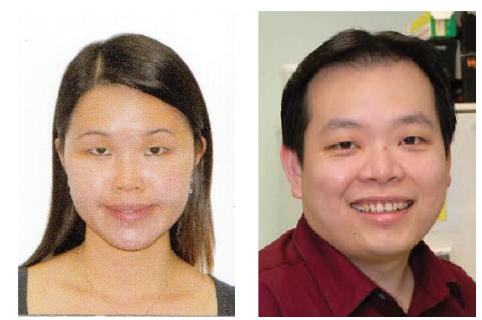

“... in vivo studies have provided us with

invaluable information to help

understand ... Chikungunya virus

infections and enable the testing of

novel therapeutic interventions."

\section{Li Sze Yeo' \& Justin Jang Hann Chu*1}

'Laboratory of Molecular RNA Virology \& Antiviral Strategies, Department of Microbiology, Yong Loo Lin School of Medicine, National University Health System, 5 Science Drive 2, National University of Singapore, 117597, Singapore

*Author for correspondence: Tel.: +65 65163278 = Fax: +65 67766872 m justin_chu@nuhs.edu.sg

The re-emergence of Chikungunya virus (CHIKV), an Alphavirus that causes debilitating fever, nausea, headache and polyarthralgia in humans, is responsible for major disease outbreaks in many regions and rekindled interest in studying the pathophysiology of CHIKV infection. There is currently no effective antiviral or vaccine against this disease. Mouse models of CHIKV infection have been developed to investigate CHIKV pathogenesis; however, each model presents both advantages and disadvantages. Nevertheless, in vivo studies have provided us with invaluable information to help understand the mechanism of CHIKV infections and enable the testing of novel therapeutic interventions.

\section{'The lack of appropriate models that accurately replicate human disease and physiology has no doubt contributed to \\ the high failure rate (50\%) of investigational new drugs in Phase II clinical trials."}

CHIKV is a positive-sense ssRNA virus, belonging to the family of Togaviridae, genus Alphavirus. It was first isolated in Tanzania, Africa in 1952 [1] and mainly transmitted by Aedes mosquitoes, Aedes aegypti and Aedes albopictus [2,3]. CHIKV can cause diseases in human characterized by fever, nausea, rash, headache, myalgia and acute/persistent polyarthralgia $[4,5]$. In rare cases, neurological complications can be observed in neonates as well as in adults [6,7]. In recent years, the re-emergence of CHIKV has led to major outbreaks in many regions [8,9], which can result in increasing social and economic burdens. Currently, there are no known vaccines or specific treatment regimens available for CHIKV infection [10].

Ideally, to obtain a more precise understanding of host-virus interaction and disease pathophysiology, clinical research studies in CHIKVinfected patients are recommended. However, research studies in humans are hampered by ethical issues and difficulties in acquiring human tissues for downstream investigation. To overcome this limitation, mouse models of CHIKV infection developed to gain further insights into CHIKV disease pathogenesis and evaluate potential novel antiviral interventions. A major advantage of using mouse models is the ability to obtain tissue samples at various time-points postinfection ( $\mathrm{pi}$ ) from different mice of the same genetic background to better understand the pathogenesis of CHIKV.

Mice of different genetic backgrounds and ages were used by various research groups to generate mouse models of CHIKV infection. Most of the models exhibited CHIKV tropism in muscles and joints similar to those observed in humans. $\mathrm{A}$ study by Couderc et al. demonstrated that 6-dayold and 9-day-old wild-type (WT) C57BL6/J mice, when infected intradermally (id.) in the ventral thorax, showed muscle weakness leading to paralysis from day 6 pi. Only $50 \%$ of the 9-day-old infected mice survived, while all 6-dayold infected mice died by day 12 pi. From day 3 pi, low levels of infectious CHIKV were found in the blood, spleen, liver and brain, and higher levels of virus were observed in the skin, joints and muscle [11]. A second investigation by the same group infected 9-day-old WT C57BL6/J mice subcutaneously (sc.) in the flank region, and showed close to $60 \%$ mortality rate from day 10

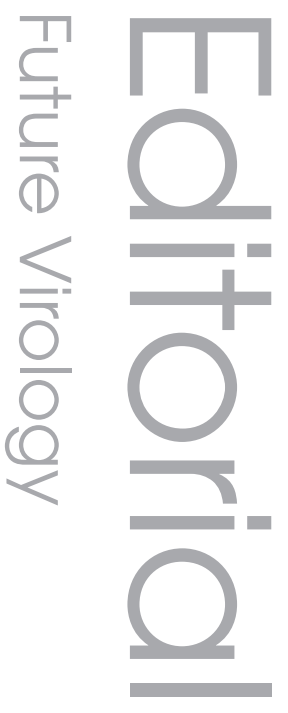

\section{Keywords}

- Alphavirus $\approx$ Chikungunya virus $=$ humanized mouse models = infection $=$ mouse models $=$ muscle weakness a pathogenesis = tropism

Future $\because$ Medicine ${ }_{\text {part of }}$ 
pi with viremia being observed in blood, muscle, liver, lung and brain as early as day 1 pi [12] Similarly, 2-3-day-old outbred mice, CD-1 and ICR mice, infected sc. with CHIKV, displayed disease symptoms such as lethargy, weight loss, dragging of hind limbs and hair loss around the site of inoculation from day 7 pi [13]. However, these symptoms were not recapitulated in adult WT mice [11,12].

\section{“6 The successful replication of disease progression in the humanized mouse model will enable the evaluation of clinical Chikungunya virus isolates with distinct host response profiles, disease outcomes or viral load properties."}

Since adult WT mice were resistant to CHIKV infection via id. and sc. routes of inoculation, immunocompromised mouse models such as adult IFN- $\alpha / \beta \mathrm{R}$ knockout mice were used to develop an in vivo adult model of CHIKV infection. It was reported in earlier studies that endogenous IFN play a critical role in controlling CHIKV infection in humans and mice. Therefore, it was hypothesized that IFN- $\alpha / \beta \mathrm{R}$ knockout mice would have increased susceptibility to CHIKV. As expected, adult IFN- $\alpha / \beta$ Rknockout mice infected via id. in the thorax manifested CHIKV-associated symptoms such as lethargy, muscle weakness of the limbs and $100 \%$ fatality at day 3 pi. These mice also exhibited viremia and histological changes in muscle, liver, joints, spleen, skin and brain [11]. Although these investigations did not accurately decipher CHIKV pathogenesis since these mice were either immunologically naive or impaired, they provided useful information on the in vivo CHIKV tropism.

Interestingly, when the virus inoculation site was changed to the ventral side of each hind foot in adult 6-week-old female WT C57BL6/J mice, typical disease outcomes including foot swelling from day $6 \mathrm{pi}$, and increased inflammatory cell infiltrates comprising monocytes, macrophages and natural killer (NK) cells were present in the foot, joint, synovial membranes, tendon connective tissues, lymph nodes, spleen and muscle. CHIKV infection also resulted in elevated virus titers in feet, muscle, liver, spleen and lymph nodes and increased levels of proinflammatory mediators such as IFN- $\gamma$, IFN- $\alpha / \beta$, TNF- $\alpha$, IL- 6 and MCP- 1 in the mouse model [14]. Likewise, a separate study by Morrison et al. showed that 2 -week-old WT

C57BL6/J mice, when inoculated in the left rear footpad exhibited swelling and edema of the left foot at day 2 pi and inflammatory changes of musculoskeletal tissues leading to arthritis, tenosynovitis and myositis [15]. However, this CHIKV-induced swelling was confined to the inoculated foot, suggesting inefficient viral dissemination in the mouse $[14,15]$. Two other studies in which CHIKV was inoculated intranasally in adult Balb/c and NIH Swiss mice exhibited weight loss and CHIKV-related pathologies such as severe spongiform changes, formation of apoptotic bodies and microglial cells in the brain, inflammatory infiltrates in lungs and severe degeneration and necrosis in the heart, liver and kidney [16,17]. Although these studies showed promising disease symptoms in adult mice, intranasal inoculation is an atypical route of CHIKV administration since CHIKV is not commonly known as an airborne pathogen. These investigations suggest that the age of the mice, site of virus inoculation and different genetic background can play important roles in the development of disease pathogenesis in the models.
6. ...the development of a humanized mouse model of Chikungunya virus
infection that mimics human disease progression will be invaluable for studying host-pathogen interaction in vivo and for evaluating antivirals and possibly vaccines."

Existing murine models for CHIKV suffer from the limitations of using neonatal mice or immunocompromised mouse models [11,13], since adult WT mice were mostly asymptomatic with sc. or id. virus inoculations [11,14]. The lack of appropriate models that accurately replicate human disease and physiology has no doubt contributed to the high failure rate $(50 \%)$ of investigational new drugs in Phase II clinical trials [18]. The humanized mouse model largely overcomes all the drawbacks inherent to the existing models. First, the NOD/SCID/Il2 $\mathrm{rg}^{-/}$ background provides a platform for studies to be conducted under physiological conditions. Second, humanized mice have a full set of human immune cells, including $\mathrm{NK}$ cells, macrophages, dendritic cells, T cells and $\mathrm{B}$ cells [19]. It is expected that the development of an in vivo platform with an intact human immune system will be ideal for investigating the roles of immune cells in the pathogenesis of 
CHIKV infection and mediating the efficacy of anti-CHIKV therapeutics.

Humanized mice have been developed by the adoptive transfer of human hematopoietic stem cells into NOD/SCID/Il2rg-/ mice $[19,20]$. These significant improvements on the model would greatly enhance elucidation of cellular and molecular basis of host-pathogen interactions. In addition, a simple method has been devised to enhance reconstitution of NK cells and myeloid cells in reconstituted mice by expressing appropriate human cytokines via the hydrodynamic injection of cytokine-encoding plasmids [20]. This has been shown to work for NK cells, monocytes, macrophages and dendritic cells, all of which could be important for CHIKV infection or the first line of defense against CHIKV infection.

Earlier studies strongly suggested that the human immune system generates more significant responses against different virus infections than mouse immune system [20]. The successful replication of disease progression in the humanized mouse model will enable the evaluation of clinical CHIKV isolates with distinct host response profiles, disease outcomes or viral load properties. The established model will also help to identify viral factors contributing to severe disease outcome. Building upon the capacity to manipulate human immune cells and gene expression in humanized mice, the role of specific human immune cells and cytokines in immune response against CHIKV virus can be investigated. Identification of immune cell types and cytokines that are important for antiviral response and disease pathogenesis could lead to the identification of targets for therapeutic development. Most importantly, the development of a humanized mouse model of CHIKV infection that mimics human disease progression will be invaluable for studying host-pathogen interaction in vivo and for evaluating antivirals and possibly vaccines.

\section{Financial \& competing interests disclosure \\ Work in the authors' laboratory is supported by the NUS Start-Up Grant (R182-000-165-133, R182-000-165- 733) and the MINDEF DIRP Grant (Project no. R182- 000-210-232, MINDEF-NUS-DIRP/2011/02). The authors have no other relevant affiliations or financial involvement with any organization or entity with a financial interest in or financial conflict with the subject matter or materials discussed in the manuscript apart from those disclosed. \\ No writing assistance was utilized in the production of this manuscript.}

\section{References}

Papers of special note have been highlighted as:

- of interest

- of considerable interest

1. Robinson MC. An epidemic of virus disease in Southern Province, Tanganyika Territory, in 1952-53. I. Clinical features. Trans. R. Soc. Trop. Med. Hyg. 49(1), 28-32 (1955).

2. De Lamballerie X, Leroy E, Charrel RN, Ttsetsarkin K, Higgs S, Gould EA. Chikungunya virus adapts to tiger mosquito via evolutionary convergence: a sign of things to come? Virol. J. 5, 33 (2008).

3. Powers $\mathrm{AM}$, Logue $\mathrm{CH}$. Changing patterns of Chikungunya virus: re-emergence of a zoonotic arbovirus. J. Gen. Virol. 88(Pt 9), 2363-2377 (2007).

4. Her Z, Kam YW, Lin RT, Ng LF. Chikungunya: a bending reality. Microbes Infect. Inst. Pasteur 11(14-15), 1165-1176 (2009).

5. Hapuarachchi HC, Bandara KB, Sumanadasa $\mathrm{SD}$ et al. Re-emergence of Chikungunya virus in south-east Asia: virological evidence from Sri Lanka and Singapore. J. Gen. Virol. 91(Pt 4), 1067-1076 (2010).

6. Robin S, Ramful D, Le Seach F, JaffarBandjee MC, Rigou G, Alessandri JL.
Neurologic manifestations of pediatric Chikungunya infection. J. Child Neurol. 23(9), 1028-1035 (2008).

7. Arpino C, Curatolo P, Rezza G. Chikungunya and the nervous system: what we do and do not know. Rev. Med. Virol. 19(3), 121-129 (2009).

8. Hochedez P, Hausfater P, Jaureguiberry S et al. Cases of Chikungunya fever imported from the islands of the south west Indian Ocean to Paris, France. Euro Surveill. 12(1), 679 (2007).

9. Lim PL, Oh HM, Ooi EE. Chikungunya in Singapore: imported cases among travelers visiting friends and relatives. J. Travel. Med. 16(4), 289-291 (2009).

10. De Lamballerie X, Ninove L, Charrel RN. Antiviral treatment of Chikungunya virus infection. Infect. Dis. Drug Targets 9(2), 101-104 (2009).

11. Couderc T, Chretien F, Schilte C et al. A mouse model for Chikungunya: young age and inefficient type-I interferon signaling are risk factors for severe disease. PLoS Pathog. 4(2), e29 (2008).

- Describes the various mouse models of Chikungunya virus (CHIKV) infection.
12. Werneke SW, Schilte C, Rohatgi A et al. ISG15 is critical in the control of Chikungunya virus infection independent of UbE1L mediated conjugation. PLoS Pathog. 7(10), e1002322 (2011).

- Describes the various mouse models of CHIKV infection.

13. Ziegler SA, Lu L, Da Rosa AP, Xiao SY, Tesh $\mathrm{RB}$. An animal model for studying the pathogenesis of Chikungunya virus infection. Am. J. Trop. Med. Hyg. 79(1), 133-139 (2008).

- Describes the various mouse models of CHIKV infection.

14. Gardner J, Anraku I, Le TT et al. Chikungunya virus arthritis in adult wild-type mice. J. Virol. 84(16), 8021-8032 (2010).

- Describes the various mouse models of CHIKV infection.

15. Morrison TE, Oko L, Montgomery SA et al. A mouse model of Chikungunya virusinduced musculoskeletal inflammatory disease: evidence of arthritis, tenosynovitis, myositis, and persistence. Am. J. Pathol. 178(1), 32-40 (2011).

- Describes the various mouse models of CHIKV infection. 


\section{Editorial Yeo \& Chu}

16. Wang E, Volkova E, Adams AP et al. Chimeric alphavirus vaccine candidates for Chikungunya. Vaccine 26(39), 5030-5039 (2008).

- Describes the various mouse models of CHIKV infection.

17. Mallilankaraman $\mathrm{K}$, Shedlock DJ, Bao $\mathrm{H}$ et al. A DNA vaccine against Chikungunya virus is protective in mice and induces neutralizing antibodies in mice and nonhuman primates. PLoS Neglect. Trop. Dis. 5(1), e928 (2011).
- Describes the various mouse models of CHIKV infection.

18. Kola I, Landis J. Can the pharmaceutical industry reduce attrition rates? Nat. Rev. Drug Discov. 3(8), 711-715 (2004).

19. Hiramatsu H, Nishikomori R, Heike T et al. Complete reconstitution of human lymphocytes from cord blood CD $34^{+}$cells using the NOD/SCID/gammac ${ }^{\text {null }}$ mice model. Blood 102(3), 873-880 (2003).
- Describes the characterization of the humanized mouse model.

20. Chen Q, Khoury M, Chen J. Expression of human cytokines dramatically improves reconstitution of specific human-blood lineage cells in humanized mice. Proc. Natl Acad. Sci. USA 106(51), 21783-21788 (2009).

- Describes the characterization of the humanized mouse model. 